\title{
A Reliability Calculations Model for Large-Scale MANETs
}

\author{
Majid Ahmad \\ Faculty of Computer Science and System Studies \\ Mewar university \\ Chittorgrah, Rajasthan.
}

\author{
Durgesh Kumar Mishra \\ Professor and Head (CSE), \\ Sri Aurobindo Institute of Technology, Indore, MP.
}

\begin{abstract}
The Mobile Adhoc Network Extensibility is an issue of concern and research. The wireless network extensibility seems to be an inherent capability of MANETs taking into consideration the Multi-hop data transfer scheme. The limits of extensibility and the tradeoff between MANET stretching and the reliability concerns need to be exercised with a deterministic model. The reliability calculation methods proposed in past have an exponential growth factor for time complexity as number of nodes increase, thus making it computationally unfeasible to calculate reliability of large scale mobile networks. This work proposes a reliability calculation scheme which is realistic for calculating reliability of large scale MANETs. The proposed approach calculates the reliability of Mobile Adhoc networks by identifying critical links within a network.
\end{abstract}

\section{General Terms}

Reliability, Wireless Networks.

\section{Keywords}

Manet, Reliability, Critical Node, Two-terminal-reliability, Scalable Networks

\section{INTRODUCTION}

The wireless technology since a decade is being pursued by industries and users as a means of advancement and usage. The wireless communication being one of the highly acclaimed areas of science and technology. As the wired had matured and emerged to be reliable far more than wireless communication, but the other advantages which wireless carry does make them more and more attractive and luring to real world applications. As the MANET applications seems to be engulfing the real world applications the peer to peer connectivity seems the absolute communication means varying different applications. Mobile Adhoc Wireless Technology (MANET) is the category of wireless networking where the flexibility in terms of Random mobility, distributed control and dynamic topologies had provided novel insight for industry \& vendors to look for. The peer to peer communication as perceived is taking a good proportion out of today's wireless technological world. MANETs have been around for a long and there usage in the areas like military, therapeutic, and other domestic applications make them a promising future prospect. The flexibilities provided by MANETs also come with some probable challenges. The concern of dynamic topology in these networks not only needs attention but up brings with other challenges also. Challenges like potential parameter variations, routing challenges, network capacity and issues of implementation are few among them. The challenges need to be taken while considering all parameters [1]. The MANET reliability does depend on various factors, and doesn't comply with the existing set standards for wired networks. The wireless reliability modeling is poles apart compared to wire line. The past work in areas of wired reliability is not capable to deal with challenges wireless provides. The important measure of wireless network reliability is terminal-pair, $\mathrm{K}$ out of $\mathrm{n}$ and all terminal reliability. The most realistic for mantes has been the two-terminal (Pair) reliability measure. Terminal-pair reliability is defined as the probability of successful communication between any two (selected) terminals in a network, so consequently terminal reliability depends on the participating terminals and also the connecting link. The reliability calculations for MANETs till recent is done by twoterminal reliability calculation model and its evolving variations, which is a very vital stride towards MANET reliability issue standardization [2]. The application of graph theory in reliability calculations can still be valuable for instant assumptions that will lead for further research. The stated approaches for computing two terminal reliability till date have a shortcoming of scalability. These approaches become computationally complex as the node number increases. The objective of this work is to define and formulate an efficient reliability calculation technique for large scale mantes.

\section{PREVIOUS WORK}

Research on wireless node reliability inherently take two terminal reliability calculation model as bedrock for reliability calculations in mobile adhoc networks. The two terminal reliability has been studied to a great extent and provide different representations by different researchers. Reliability calculation for wireless has attracted some attention by various research groups in past. Rai et al provided a basic and fundamental way of approaching reliability problem by Terminal reliability calculations for generalized computer networks [6].But the methods developed for wired networks had lot be modified to map them for wireless networks. Moreover the wireless networks also have far more variations from PCF (Point Coordination) based to DCF (Distributed Coordination) based wireless networks. Chen et al introduced end-to-end expected instantaneous reliability scheme to make reliability calculation more generic across heterogeneous environments for wireless Networks [7]. Jason et al have defined some key reliability drivers (Number of nodes, Node reliability, and link reliability) for MANETs with applicability of the previous schemes was taken as an objective in their work. They also introduced methods relying on advanced modeling approach that recognizes the effects of mobility on the formation of the network's configurations [3][4]. Thilagavathe et al have come up with another reliability computation method involving predication parameter [5]. Graphical analysis \& solutions in past have also been taken in 
area of reliability. The evaluation of various mobility models like Random-way point, City Section and Manhattan mobility was done to comparatively study those mobility models [9]. Kharbash et al developed a scheme to calculate two-terminal reliability for MANETs which is a noteworthy work till date [2].They introduced the calculation algorithm and which was more realistic than the previous developments. But still their scheme left a void by not introducing the probability factor of node location. Here in this paper we have taken care of that factor in order to achieve higher accuracy of two terminal calculations. All the network reliability techniques proposed so far has been computationally (time) efficient till the size of the network (number of nodes) is limited. The large scale network reliability calculation has left a challenge for researchers. All the techniques simulated or presented had a small size of node number to depict the reliability calculation problem. Two-terminal reliability calculation algorithms presented in past have described reliability calculation (two terminal reliability) as a \#P-complete problem. The work in [12] proposed tried to reduce the calculation into P-type (polynomial time). The complexity of any algorithm for twoterminal reliability calculation is proportionate to the complexity of given configuration of that network. Sheng et al came up with a novel algorithm of critical node detection with high detection accuracy [10]. The node is critical if its sudden failure divide a network in to two disjoint sub networks. Thus network's extensibility does somehow depend on the criticality of these critical nodes. The area of extension of a network can be increased by taking care of these critical nodes. The work on MANET reliability has been done to some extent in past but to make it more suitable to different kind of applications need to be studied. The current research is been done on the link, node \& link-node with primary goal of leveraging the benefits from past research on Wireless scenario in general and studying the scalability issue of MANETs

\section{Reliability Fundamentals.}

A scalable MANET network is to be defined as a network which will keep on stretching as the hops are added on. As MANETs support multi-hop communication, making it easy to realize a scalable network. Multi-hop communication but has certain challenges like the routing burden on intermediate nodes, the effect of extended network on the power consumption of intermediate nodes, bandwidth fairness of the channel and so on. The above challenges do provide a better insight into reliability aspect of scalable networks. This work calculates the two terminal reliability of MANET configurations, and then suggests a better computationally efficient approach which is more realistic for scalable mobile ad-hoc networks by identifying critical links within a network. A MANET can be realized as a sequence of a number of NLNs. Here we introduce a term NLN (Node-Link-Node), and where every NLN has a probability factor associated. Nodes in NLN have some pre specified independent working/operational probability. Respectively, the Link existence probability also adds to the NLN probability. The probability of link cannot be pre-specified and is dependent on operational status of connecting nodes. Furthermore a link is operational only when two participating nodes are not only operating but do reside in each other's transmission range. ,but the conditions that two nodes happen to reside in each other's range is a factor of concern for Mobile nodes and some probability can be forecasted in advance. The probability that communicating nodes happen to be in each other's range can be calculated from the node behavior (Maximum velocity, boundary limits etc). Assume that the probability be $r_{t}$

$$
0<=r_{t}<=1
$$

The probability of existence of a link Le $e_{\text {Prob }}$ is the probability that they reside in each other's range. Thus $\mathrm{Le}_{\text {prob }}$ for any possible NLN is

Le $e_{\text {prob }}=r_{t}$

The overall probability of any NLN is

$$
N L N_{\text {Prob }}=N 1_{\text {Prob }} \mathrm{N} 2_{\text {Prob }}\left(\text { Le }_{\text {Prob }}\right)
$$

Where $\mathrm{N} 1_{\text {Prob, }} \mathrm{N} 2_{\text {Prob }}$ is the operational probability of two nodes forming a possible NLN and $\mathrm{Le}_{\text {Prob }}$ is the probability of existence of a link. A network may in total comprise of

$$
\mathrm{e}=\mathrm{n}(\mathrm{n}-1) / 2
$$

number of possible NLN sequences, reliability of a particular Configuration (State) is the product of all possible individual NLN probabilities

$$
\operatorname{Conf}_{\text {Prob }}(t)=\prod_{1}^{e} \quad\left(\mathrm{NLN}_{\text {Prob }}\right)^{\mathrm{Lt}}\left(1-\mathrm{NLN}_{\text {Prob }}\right)^{1-\mathrm{Lt}}
$$

Where $\mathrm{Lt}=1$ if the link exists in the current NLN otherwise $\mathrm{Lt}=0$.

$$
\sum_{i=1}^{E} \text { Conf }_{\text {Prob }}=1
$$

is the sum of all configuration probabilities Where $E=2^{e}$ Now calculating the overall 2-terminal reliability of the network for any two nodes (Sender, Receiver) we have

$$
\text { 2TR }_{\mathrm{S} \rightarrow \mathrm{R}}=\sum_{i=1}^{E} \operatorname{Conf}_{\text {Prob }} \mathrm{P}_{\mathrm{i}}
$$

Where $P_{i}=1$ if at least a single path exists between selected sender and receiver otherwise $\mathrm{Pi}=0$.

An existing scheme for two-terminal reliability calculation has been introduced in [6]. This method calculates the operational paths by depicting a tree structure; the limitation with the scheme is that as the node size increases the structure seems to be difficult to manage and calculations exponentially increasing. Thus the method is suitable to environments which are limited to small node number size. We are here going to provide a method which is more realistic for extensible (Large sized) Mobile Adhoc Networks. As conceived that, not all node links exist with same probability. The nodes which are close in proximity create a high probability of link formation than nodes which are n-hops apart. We are taking into consideration the fact that nodes form and reform links but with high probability in close neighborhood. A node does not follow a normal probability distribution of link formation for 
all the nodes within a single network. Manets generally have a peer to peer communication scenarios and seldom exhibit unique multiple redundant paths between intended sender and receiver, thus presence of a so called critical node is almost certain. Moreover a Manet generally is realized as a network comprising of different node classes [11]. A configuration which includes a critical node (critical NLN) in its sequence is prone to higher load leading to factors (higher battery consumption etc) of higher probability of node (link) failure too. Figure 1 shows us a network configuration where nodes 3, 4 form a critical NLN. The NLN formed by these nodes can be described as a critical NLN, which if failed does make communication from S1 to R6 clogged. Every communication path from node S1 to node R6 has the link between node 3 and node 4 as common, thus the communication from node $\mathrm{S} 1$ to node R6 can be realized in three parts. One the communication from node1 to node3 (sub-network SN1).Second communication from node3 to node4 (critical NLN). Third communication from node4 to node6 (subnetwork SN2). The configuration in figure 1 is an example of low level redundancy, thus calculations are simpler than highlevel redundancy. A high level redundancy has higher failure rate than a low level redundancy, hence configurations with low level redundancy is desirable than high level redundancy. Every individual communication (two terminal) is multiplied to the overall reliability of the network as whole. Now in a state of a network we can apply an existing algorithm like in [10] for detecting global critical nodes in a network. The reliability calculations of a network can be further fine tuned by including the critical NLN as a parameter. Now we introduce a scheme where a state having one or more critical links will make reliability calculation more efficient in terms of computational time, hence will prove attuned with large scale manets.

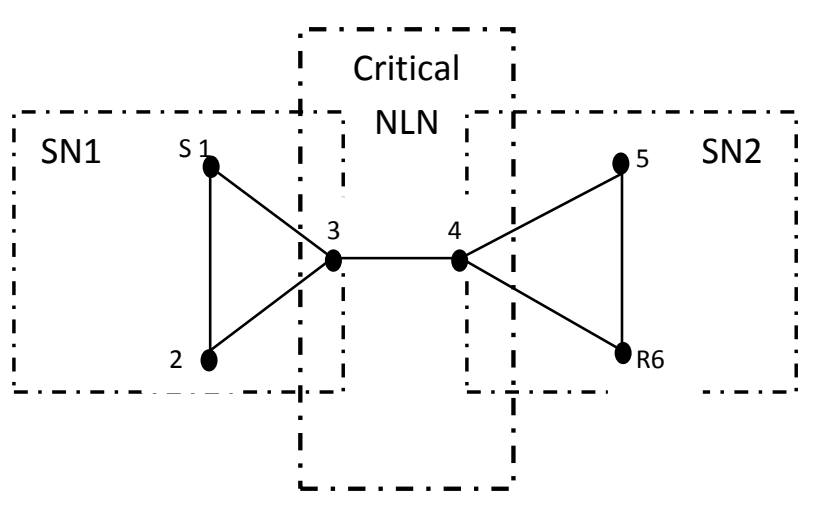

Figure1

\section{Proposed method}

\subsection{Assumptions.}

Here we assume to have a network represented by a graph $\mathrm{G}(\mathrm{V}, \mathrm{E})$. The reliability calculation of the network at any point in time depends on the configuration state at that point. We define a critical NLN as the link which is common to all operational paths between intended sender and receiver node at that moment of time. Thus that presence of any critical
NLN will divide the network into two sub-networks. The process of dividing the network into sub-networks will continue till last critical NLN is done. We are also modifying the usage of critical node detection algorithm proposed in [10] to suite the NLN critical link detection. We are enumerating all the network nodes with sender node as first and the destination as the last node (usage of any routing algorithm can modify the enumeration accordingly).

\subsection{Introduced Algorithm:-}

Step1. Using algorithm in [10] identify whether the current node is forming a critical NLN. Step2. If NLN not critical \& NLN not including destination node move on next node (enumerated order), go to $\quad$ Step1. Step3. If NLN critical, calculate the Total two terminal reliability from source node up to the first node of the current NLN (sub-network).

Step4. Start again with second node of the current NLN as source, go to step1.

Step5. Multiply all the individual sub-network reliabilities and the individual reliabilities of all critical NLNs to calculate the final reliability of this network configuration.

\subsection{Description:}

The algorithm starts with the sender node, and ends up to the destination node. Every selected node $\mathrm{n}_{\mathrm{i}}$ is checked if forming a critical node. If it's not a critical node we move on $\mathrm{n}_{\mathrm{i}+1}$. The node if forming a critical node is assumed to be the destination node of this sub-network till now. The overall two terminal reliability is calculated from the source to this current node. Thus we have calculated the two-terminal reliability up to this node (sub-network). We start from the other side of the NLN and repeat the above process till we don't reach the final destination. Every critical link will divide a network into two sub-networks and the two sub networks will have $\mathrm{i}$ and $\mathrm{n}$ - $\mathrm{i}$ nodes. As the two sub networks can further be divided recursively till the whole network gets exhausted. After calculating the reliability of each sub network we product the reliability of all the sub networks and critical NLNs. This method is computationally efficient as the overall reliability can be calculated in parallel (each sub network) if algorithm is modified. This algorithm suits better for large scale networks like MANETS and does reliability calculation effectively.

\subsection{Algorithm evaluation}

The reliability calculations for large network are computationally very harsh and as the MANETs change on configurations dynamically the reliability calculation becomes more complex in nature. We are introducing the above algorithm which takes the scalability issue in concern while calculating reliability for large MANETs. A comparison can be done between the classic techniques and the one proposed here. A MANET with $n$ nodes in total will have all of $2^{\mathrm{n}(\mathrm{n}-1) / 2}$ configurations and the calculation for all the configurations is to be done before the final reliability is calculated. The proposed algorithm makes calculation less complex by 
reducing the computational iterations. A MANET with at least one critical link will reduce the computational complexity to

$$
\left.\mathrm{C}=2 \underset{\mathrm{i}=2}{\mathrm{n}-1} \sum^{\mathrm{i}(\mathrm{i}-1) / 2}\right) / \mathrm{n}-1 .
$$

The above equation describes tne compıexity of reliability calculation for a manet having one critical link. A Manet having more than one critical link will divide a network substantially into large number of sub networks. Thus taking the above equation in consideration, a network having $\mathrm{m}$ number of critical links among total of $n$ number of nodes can reduce the complexity to an extent which can be recursively calculated as

$$
c(n, m)=\left[\sum_{i}^{n-1}(c(i, m-1)+c(n-i, m-1))\right]
$$

Where $c(n, 0)=2^{n(n-1) / 2} \quad$ (Not a single link is critical) $\mathrm{c}(1, \mathrm{~m})=0 \quad$ (Only one node left) $\mathrm{c}(\mathrm{n}, \mathrm{m} / \mathrm{n}=\mathrm{m}+1)=0 \quad$ (number of nodes equal to number of critical links).

The average case for total number of configurations can be calculated by

$$
\mathrm{C}=\mathrm{c} / \mathrm{n}-1 \mathrm{c}_{\mathrm{m}}
$$

As total $m$ numbers of links are to be selected from $n-1$ number of nodes.

\subsection{Complexity Calculation}

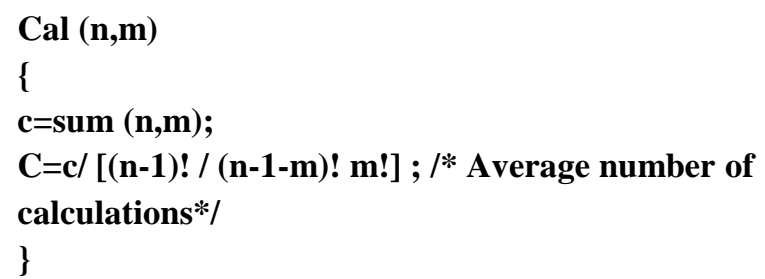

\section{CONCLUSION}

We have presented a reliability calculation technique that is more efficient than the existing methods of reliability calculations. As all the previous stated techniques have a high exponential increase in calculation complexity as the node number increase, but this algorithm provides a control by introducing critical NLNs. Reliability calculation is recursively done and proves to be an efficient calculation technique. Reliability calculation is necessary so that to mitigate after failure effects and for large scale networks this is more challenging.

\subsection{FUTURE WORK}

Our current work is about reliability calculation, where a single critical NLN reduces the complexity and provides a much improved calculation method. Future work will be about introduction of more than one critical NLN.

\subsection{RESULT SET DISCUSSION}

The results in figure2a drawn from implementation of this algorithm shows how reliability calculation complexity is reduced and reliability calculation becomes realistic for large scale networks, which till date is a major challenge. The proposed scheme takes critical nodes as the calculation assumptions and thus this method should be able to limit reliability calculation complexity within practical reach. Also figure $2 b$ show that increase in node reliability has an higher impact when existing reliability of the system crosses $60 \%$. A slight increase in average node reliability sharply increases the overall reliability of a network.

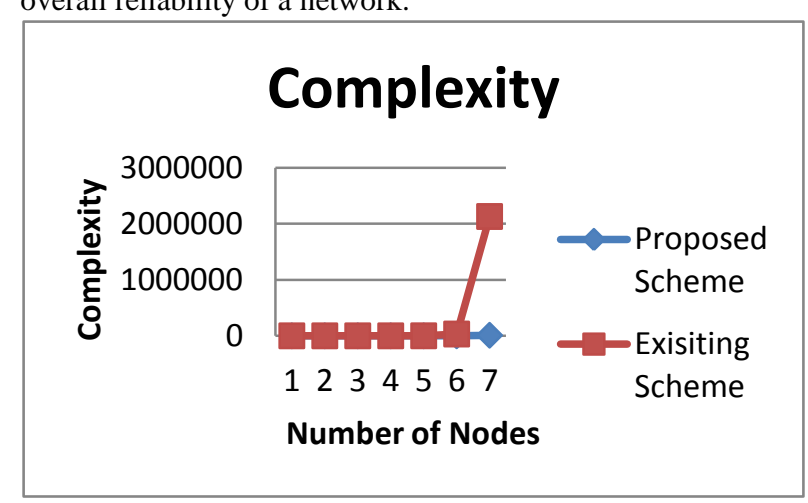




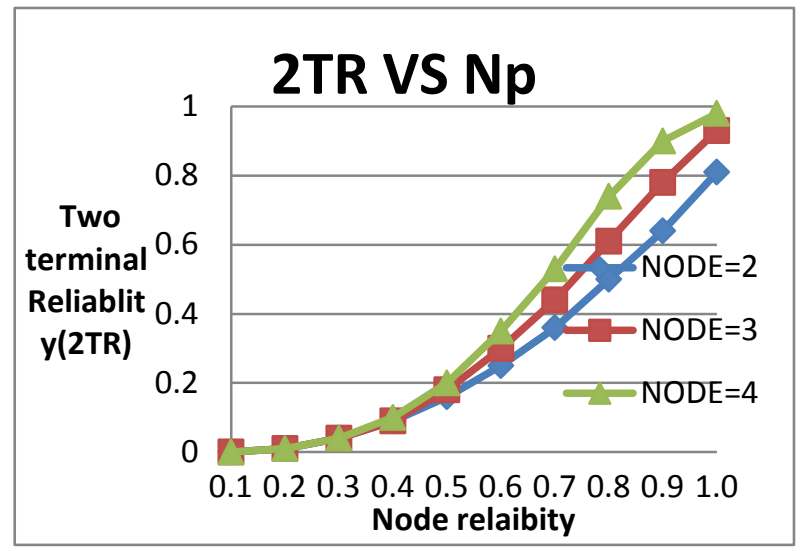

Figure 2b

\section{REFERENCES}

[1] "Analyzing the manet variations, challenges, capacity and protocol issues", G. S. Mamatha, and Dr. S. C. Sharma, International Journal of Computer Science \& Engineering Survey (IJCSES) Vol.1, No.1, August 2010.

[2] "Computing two-terminal reliability in mobile ad hoc networks", S. Kharbash and W. Wang, Wireless Communications and Networking Conference, 2007.WCNC 2007. IEEE, 11-15 March 2007.

[3] "Recent Research on the Reliability Analysis Methods for Mobile Ad-hoc Networks", Jason L. Cook, Picatinny Arsenal Jose Emmanuel Ramirez-Marquez, Systems Research Forum, 2007, Vol. 2.

[4] "Mobility and reliability modeling for a mobile ad hoc network", Jason L. Cook, Jose Emmanuel RamirezMarquez,IIE transactions, Volume 1, 2008.

[5] "Prediction Based Reliability Estimation in Manets with Weibull Nodes", V. Thilagavathe, K. Duraiswamy, European Journal of Scientific Research ISSN 1450216X Vol.64 No.2 (2011), pp. 325-329 (C) EuroJournals Publishing, Inc. 2011

[6] "Computing Terminal Reliability of Computer Network, Reliability Engineering",S. Rai, A. Kumar, and E. V. Prasad, vol. 16, no. 2, pp. 109. 119, 1986
[7] "Reliability analysis for various communication schemes in wireless CORBA",X. Chen and M. R. Lyu, IEEE Transactions on Reliability, vol. 54, no. 2, pp. 232.242, June 2005.

[8] "Calculation of node-pair reliability in large networks with unreliable nodes. IEEE Transactions on Reliability"Torrieri, Don. 1994, Vol.43, no.3: 375-377.

[9] "On the probability of k-connectivity in wireless ad hoc networks under different mobility models",Natarajan Meghanathan and Sireesha Gorla,, International journal on applications of graph theory in wireless ad hoc networks and sensor networks (GRAPH-HOC) Vol.2, No.3, September 2010

[10] "Critical Nodes Detection in Mobile Ad Hoc Network",Min Sheng, Jiandong Li and Yan Shi,", IEEE Computer Society 2006.

[11] "Extended Multi-Class Approach for Improvement in Overall Throughput Efficiency of Mobile Adhoc Networks", Dr. W. U. Khan and Majid Ahmad International Journal of Computer Theory and Engineering, Vol. 3, No. 3, June 2011.

[12] AboElFotoh, H.M.; Colbourn, C.J.; , "Computing 2terminal reliability for radio-broadcast networks," Reliability, IEEE Transactions on , vol.38, no.5, pp.538555, Dec 1989.

[13] Egeland, G.; Engelstad, P."The availability and reliability of wireless multi-hop networks with stochastic link failures," Selected Areas in Communications, IEEE Journal on , vol.27, no.7, pp.1132-1146, September 2009

\section{AUTHOR'S PROFILE}

Durgesh.K.Mishra has done $\mathrm{PhD}$ (Computer Engineering). He is a senior member of IEEE.He is having around 21 Yrs of teaching experience and more than 6 Yrs of research experience. Presently he is working as Professor (CSE) and Dean (R\&D) in Sri Aurobindo Institute of Technology, Indore, India. He is also a Visiting Professor at IIT Indore.

Majid Ahmad is a $\mathrm{PhD}$ scholar, working in the area of MANET reliability. His past research has been in the area of MANET protocol tuning. He has three years of research \& Teaching experience. 